\title{
ENCONTROS DE ARACNÓLOGOS DO CONE SUL (1997 - 2003), UM INVESTIMENTO DE SUCESSO
}

\author{
Antonio D. Brescovit ${ }^{1}$, Cristina Anne Rheims ${ }^{12}$ \& Glauco Machado ${ }^{3}$
}

Recebido em 07/12/2003

Publicado em 01/02/2005

Biota Neotropica v5 (n1a) - http://www.biotaneotropica.org.br/v5n1a/pt/abstract?point-of-view+bn022051a2005

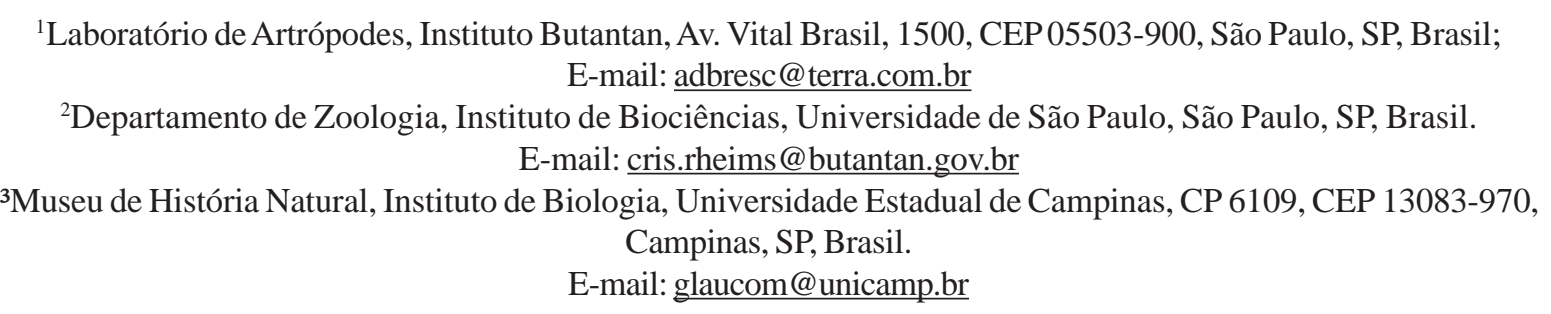

\begin{abstract}
Here we present a brief history of the "Encontros de Aracnólogos do Cone Sul (EACS)”, a meeting of mostly South American arachnologists held every two years since 1997. Data compiled from the abstract books show how these meetings have contributed towards the development of South American arachnological research.
\end{abstract}

Key words: Encontro de Aracnólogos do Cone Sul, history, arachnology, South America.

\section{Resumo}

Neste texto apresentamos um breve histórico dos Encontros de Aracnólogos do Cone Sul (EACS), uma reunião de aracnólogos sul americanos realizada a cada dois anos desde 1997. Os dados foram obtidos a partir dos livros de resumos e mostram a importância dessas reuniões para o desenvolvimento da pesquisa em aracnologia na América do Sul.

Palavras-chave: Encontro de Aracnólogos do Cone Sul, histórico, aracnologia, América do Sul.

http://www.biotaneotropica.org.br 


\section{Histórico}

Os Encontros de Aracnólogos do Cone Sul (EACS) foram concebidos com o objetivo de gerar uma oportunidade de intercâmbio científico e acadêmico, discutir temas gerais em aracnologia e propiciar colaborações entre pesquisadores de diferentes países. O esforço de reunir aracnólogos latino-americanos foi iniciado pelos uruguaios em dezembro de 1997, na cidade de Montevidéu (veja resenha sobre o IV EACS em Acosta, 2003). No entanto, duas edições de uma fase embrionária dessa reunião já haviam sido realizadas no Brasil em 1996 e 1997. Os aracnólogos brasileiros se reuniram na Serra do Japi, Jundiaí, SP, para o que chamaram de Encontro de Aracnologia (Pinto da Rocha, 1996; 1997).

A primeira edição do evento em Montevidéu contou com a presença de 40 aracnólogos da Argentina, Brasil, Peru e do país sede. A receptividade que o I EACS encontrou entre os pesquisadores sul-americanos mostrou que a maturidade científica da comunidade demandava a continuidade do evento a fim de possibilitar uma maior integração entre profissionais e estudantes de aracnologia. Neste sentido, em 1999 foi realizado na cidade de Porto Alegre, Rio Grande do Sul, Brasil, o II EACS. O número de participantes aumentou muito, especialmente entre os estudantes de graduação e pós-graduação, com um incremento expressivo no número de trabalhos apresentados.

O III EACS, realizado em 2002 na cidade de Córdoba, Argentina, apresentou algumas novidades em relação às edições anteriores. Pela primeira vez na história do evento formou-se uma Comissão Científica para a avaliação dos resumos submetidos, o que, certamente, contribuiu para a excelência da programação científica. O III EACS também inovou com a criação do Concurso de Jovens Aracnólogos, uma tentativa bem sucedida de prestigiar a participação de estudantes que apresentaram trabalhos. A realização do III EACS foi, sem dúvida, a consolidação do encontro como o mais importante evento no calendário dos aracnólogos sul-americanos.

Após o IV EACS, realizado em dezembro de 2003 na cidade de São Pedro, SP, parece interessante apresentar uma síntese dos quatro encontros realizados até o momento. Os Encontros de Aracnólogos do Cone Sul (EACS) são hoje a maior reunião de aracnólogos na América do Sul e, apesar de recentes quando comparados aos encontros realizados nos Estados Unidos, Europa e mesmo no Japão, já superam estes em número de participantes e de trabalhos apresentados (Tabela 1). O grande número de participantes e a crescente importância das reuniões para a aracnologia sul-americana elevaram os encontros ao nível de congresso, de modo que em 2005 será realizado o I Congresso LatinoAmericano de Aracnologia, sediado no Uruguai.
Nesta síntese mostramos a evolução dos EACS, enfocando o crescimento no número de participantes, a diversidade dos mesmos no continente sul-americano, o incremento de trabalhos apresentados e a presença já significativa de aracnólogos de outros continentes. As informações para esta sinopse foram compiladas dos quatro livros de resumos, publicados em 1997, 1999, 2002 e 2003.

\section{Participantes com resumos}

A Figura 1 mostra, em linhas gerais, a origem dos participantes que apresentaram resumos nas quatro edições do EACS, dando ênfase para os quatro países com maior número de participantes. No item “Outros” estão incluídos pesquisadores oriundos do Peru, Colômbia, Costa Rica, Cuba, Venezuela, México, Estados Unidos, Alemanha e Espanha).

Em todos os encontros houve predominância de participantes brasileiros, seguidos pelos argentinos e uruguaios, respectivamente, uma vez que estes países abrigam os maiores centros de pesquisa em aracnologia da região neotropical. A representatividade do Brasil nos encontros é bastante expressiva, com a participação de 18 pesquisadores no I EACS. Nas três edições seguintes a participação dos brasileiros foi de 44, 22 e 107, respectivamente. Em geral, o maior número de participantes brasileiros foi registrado em eventos realizados no próprio país. A Argentina e o Uruguai aparecem logo atrás do Brasil, com uma participação de seis a 12 pesquisadores por evento. É interessante salientar que a Colômbia, ausente nos dois primeiros encontros e representada por apenas um pesquisador no terceiro, esteve presente no IV EACS com uma comitiva de 10 pessoas. Esse número praticamente equivale aos apresentados por Argentina e Uruguai, sugerindo que a aracnologia está em franca expansão na Colômbia.

A presença de especialistas de outros continentes também merece destaque. No I EACS apenas um colega proveniente dos Estados Unidos estava presente. Já no último encontro, estavam presentes pelo menos cinco aracnólogos oriundos dos Estados Unidos, Espanha e Alemanha, entre participantes e convidados. A presença desses pesquisadores mostra que o evento já vem chamando atenção de especialistas de outros continentes.

\section{Resumos apresentados}

Nota-se que a cada EACS houve um incremento no número de trabalhos apresentados (Figura 2), com exceção apenas do III EACS, no qual se observa uma pequena redução no número de resumos. Em Montevidéu foram apresentados 43 resumos, em Porto Alegre foram 89, em Córdoba foram 71, culminando com 201 em São Pedro.

Dentre os quatro países melhor representados destaca-se o Brasil com maior número de trabalhos nos quatro eventos. No IV EACS a produção brasileira chega a três vezes o total do que foi apresentado nas edições 


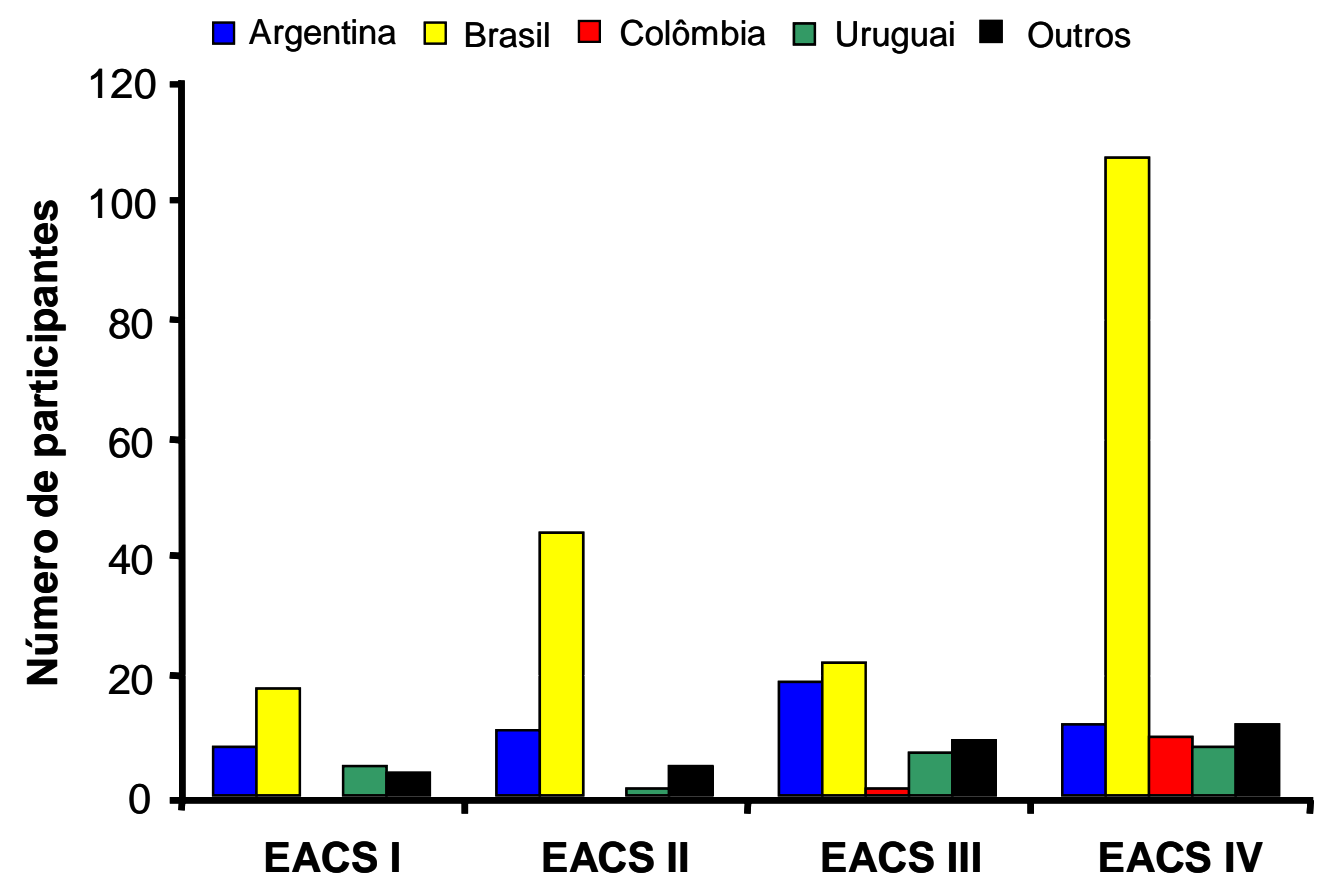

Figura 1. Número de participantes nos quatro Encontros de Aracnólogos do Cone Sul.

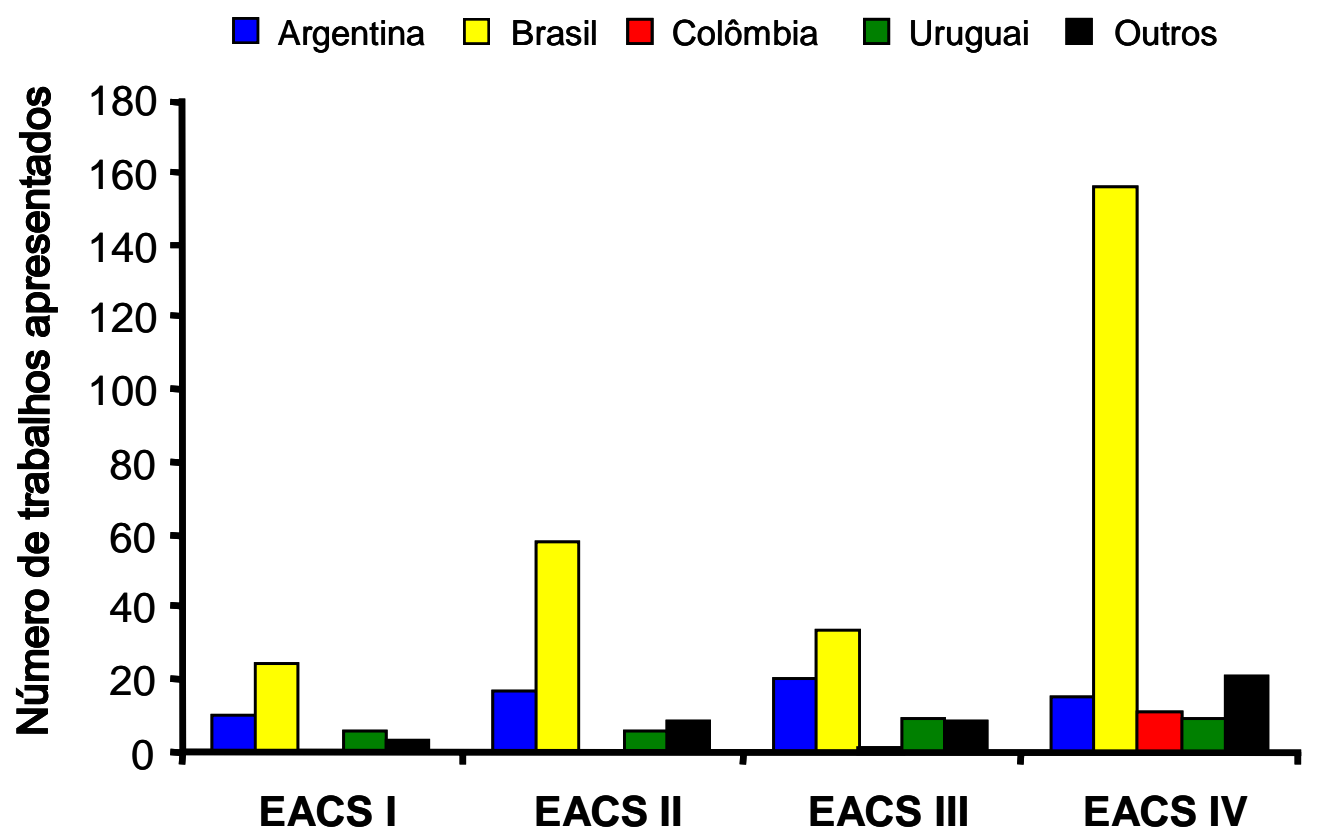

Figura 2. Número de resumos apresentados durante os quatro Encontros de Aracnólogos do Cone Sul. 


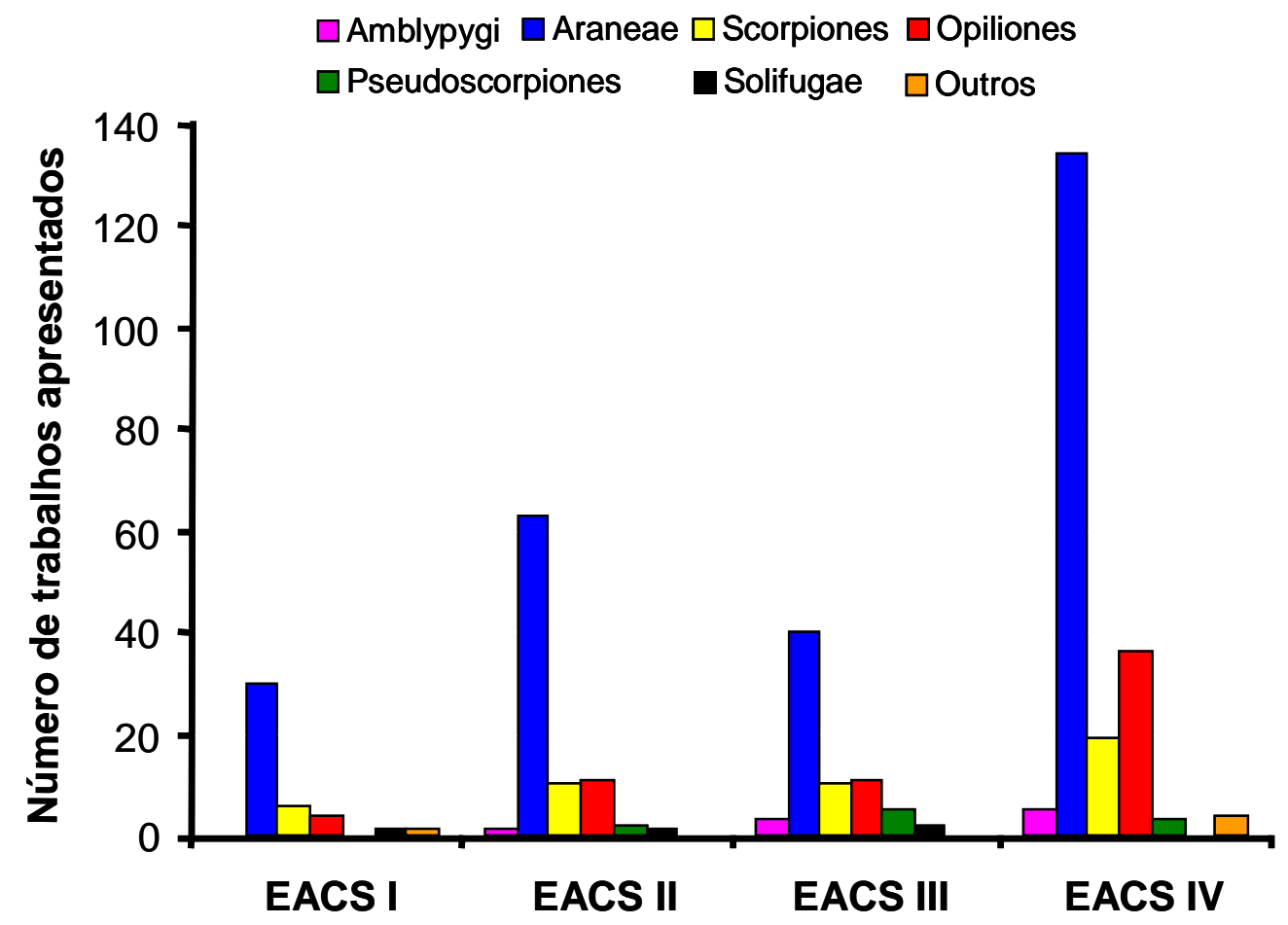

Figura 3. Ordens de Arachnida abordadas durante os quatro Encontros de Aracnólogos do Cone Sul.

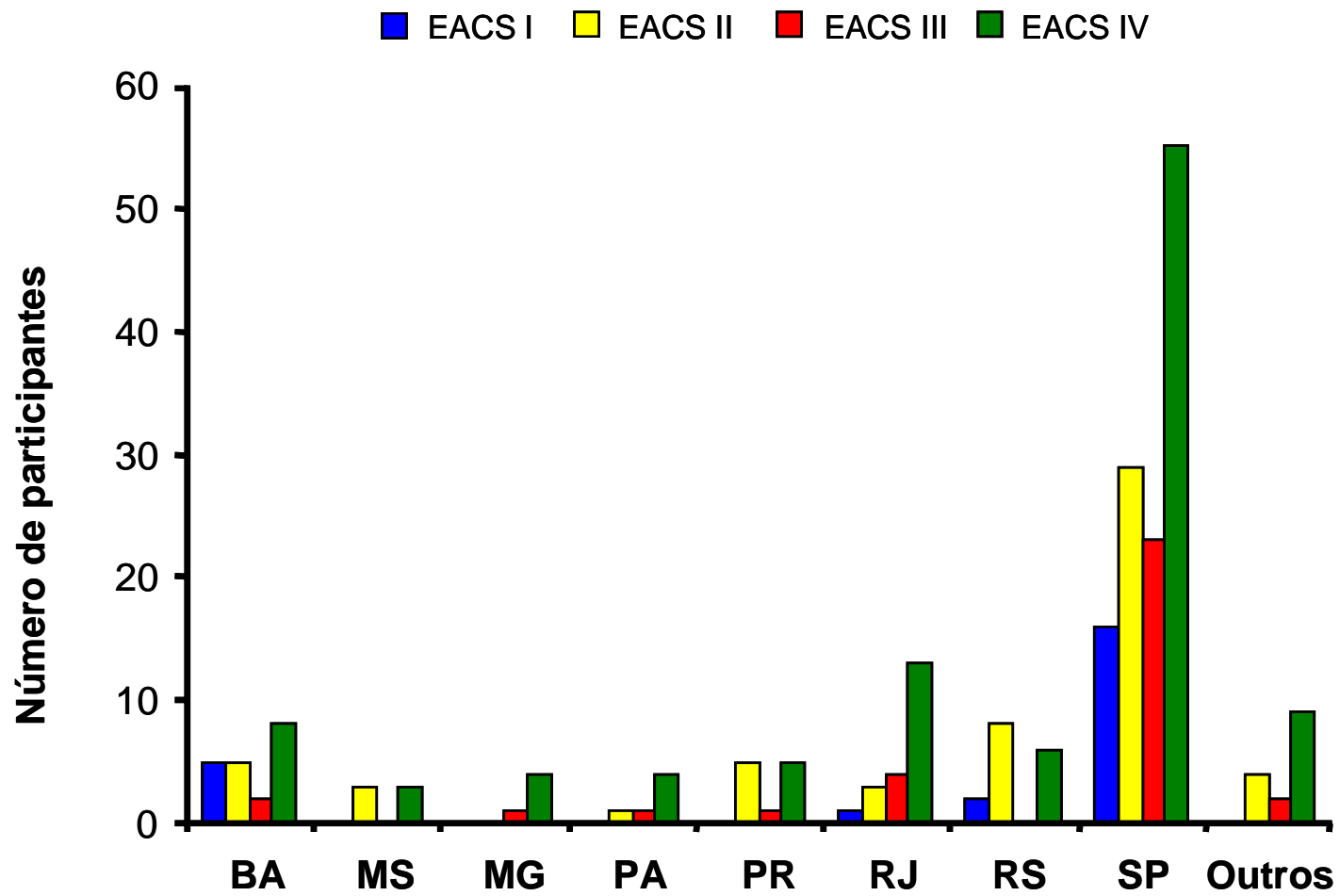

Figura 4. Número de participantes do Brasil nos quatro Encontros de Aracnólogos do Cone Sul. 
Tabela 1. Quadro comparativo dos últimos quatro encontros de aracnologia na América do Sul, Europa e Estados Unidos.

\begin{tabular}{ccccc}
\hline Edição & Ano & $\begin{array}{c}\text { Participantes com } \\
\text { trabalho(s) }\end{array}$ & $\begin{array}{c}\text { Resumos } \\
\text { apresentados }\end{array}$ & Fonte \\
\hline $18^{\circ}$ & \multicolumn{4}{c}{ Coloquium Europeu de Aracnologia } \\
$19^{\circ}$ & 1999 & 100 & 52 & Dunlop (2000) \\
$20^{\circ}$ & 2000 & 110 & 85 & Bell (2000) \\
$21^{\circ}$ & 2001 & 117 & 108 & Shaw (2002) \\
& 2002 & $96-100$ & 85 & Penney (2003) \\
$24^{\circ}$ & Reuniões da American Arachnological Society (EUA) & Starr (1999) \\
$25^{\circ}$ & 2000 & 80 & 60 & American Arachnology (2002) \\
$26^{\circ}$ & 2001 & 99 & 51 & Reiskind (2003) \\
$27^{\circ}$ & 2002 & 120 & 65 & Cushing (2003) \\
& 2003 & 140 & 94 & - \\
I & 1997 & Encontro de Aracnólogos do Cone Sul & - \\
II & 1999 & 69 & 43 & Acosta (2002) \\
III & 2002 & 63 & 71 & Machado \& Brescovit (2003) \\
IV & 2003 & 149 & 201 & \\
\hline
\end{tabular}

anteriores e, no evento, a produção é de $77 \%$ do total de resumos apresentados. Na seqüência vêm o Uruguai e a Argentina, com uma média de 8 e 18 trabalhos por evento, respectivamente. Mais uma vez a Colômbia merece destaque com 11 resumos no IV EACS.

\section{Ordens de aracnídeos abordadas}

Todas as ordens de Arachnida já foram abordadas nos encontros, à exceção dos Palpigradi que talvez seja a ordem mais enigmática dentro da classe. O I EACS teve inclusive um trabalho apresentado com a ordem Acari (Figura 3). Essa ordem é, em geral, abordada em congressos de acarologia, cujos especialistas normalmente não participam dos congressos de aracnologia.

A ordem que concentra o maior número de trabalhos apresentados em todos os encontros é Araneae, representando sempre mais de $50 \%$ do total de trabalhos. Seguem com grande número de trabalhos as ordens Opiliones, com 62 resumos, e Scorpiones, com 45 resumos. Essas duas ordens seguem crescendo em abordagens desde o primeiro encontro e no IV EACS o número de trabalhos apresentados sobre essas duas ordens já supera em mais de duas vezes o que foi publicado nos demais encontros. Com relação às demais ordens, o número de trabalhos é muito baixo, não ultrapassando 10 resumos nos quatro encontros. Cabe notar que certas ordens como Ricinulei e Thelyphonida só estão representadas no IV EACS.

É de se esperar que a ordem Araneae seja abordada pela maioria dos trabalhos. O histórico da aracnologia nas instituições sul-americanas mostra que o número mais expressivo de especialistas foi formado na pesquisa com aranhas. A América do Sul carece de especialistas nas chamadas "pequenas ordens" de Arachnida. Os dados desta sinopse mostram que só agora estão sendo formados especialistas em Amblypygi e Pseudoscorpiones, e que as demais ordens, como Ricinulei, Thelyphonida e Solifugae, embora representadas por alguns trabalhos, são abordadas esporadicamente. Em geral, estas ordens são trabalhadas por especialistas de outras ordens, algo que seria de se esperar, considerando também a baixa diversidade e/ou ocorrência desses grupos no subcontinente. 


\section{Presença do Brasil nos eventos}

Como o Brasil é o país com maior presença e produtividade em todos encontros, algumas considerações específicas são interessantes. Na Figura 4 destacam-se os três estados que sempre estiveram presentes nos eventos: São Paulo, Rio de Janeiro e Bahia. Presentes em pelo menos três encontros destacamos os representantes do Rio Grande do Sul, Paraná e Pará.

Em relação à proporção de participantes, o estado de São Paulo lidera em todos os encontros. Cabe destacar aqui que os maiores grupos de pesquisa e centros formadores de aracnólogos do país estão no estado de São Paulo. Além disso, o IV EACS foi realizado em São Paulo, facilitando a presença de um grande número de alunos de graduação que, em geral, não têm condições de participar de eventos realizados em outros países ou estados muito afastados da região sudeste.

A Figura 4 mostra também um crescimento contínuo da presença do Rio de Janeiro, onde se destaca pelo menos um grande centro formador de aracnólogos, o Museu Nacional. Outros três estados merecem destaque, dos quais o que tem apresentado maior formação de aracnólogos é a Bahia. Apesar de ter apenas um centro em aracnologia, a Universidade Federal da Bahia, o estado possui muitos pesquisadores que fizeram pós-graduação em outras regiões e retornaram a este para trabalhar, tornando-o um dos pólos mais expressivos da região nordeste. Outro caso de rápido incremento de pessoal é o Paraná que, em função dos problemas relacionados aos acidentes com aranhas Loxosceles, viu seu quadro de aracnólogos crescer bastante nos últimos anos. Finalmente, merece destaque o Pará, que iniciou sua participação na aracnologia nacional com a contratação de um sistemata de aranhas pelo Museu Paraense Emílio Goeldi. Da mesma forma que o Pará, a formação de um grupo de aracnologia em Manaus, na Universidade Federal do Amazonas, deve estimular os estudos com o grupo no Amazonas e, no futuro, esperamos contar com a presença constante de pesquisadores deste estado.

\section{Comparações com outras reuniões aracnológicas}

Apesar da história recente, pode-se fazer um paralelo entre os EACS e as reuniões realizadas na América do Norte e na Europa, continentes onde já existe uma longa tradição de encontros aracnológicos (Tabela 1). É possível notar que no IV EACS o número de participantes é similar ao das reuniões aracnológicas realizadas nos últimos quatro anos na América do Norte e Europa. Nota-se também que, em 2003, o IV EACS suplantou os encontros europeus e norte-americanos em número de resumos apresentados. Em relação às reuniões norte-americanas, o maior diferencial é que estas são compostas em sua maioria por especialistas dos Estados
Unidos, enquanto que nos EACS e nos eventos europeus há uma maior diversidade de países representados.

Uma tradição dos encontros europeus de aracnologia é a publicação de anais das reuniões, que ocorre em volumes próprios ou agregados a alguma revista científica de ampla circulação. Nos encontros sul-americanos essa iniciativa surgiu apenas no IV EACS, com a proposta de publicação de trabalhos na íntegra em uma revista eletrônica, a BIOTA NEOTROPICA. A idéia era dar uma maior visibilidade aos EACS, possibilitando a apresentação dos resumos em forma de publicação e dando a oportunidade aos aracnólogos para divulgarem seus resultados.

\section{Conclusões}

Os EACS representaram até 2003 um passo para reunir os especialistas em Arachnida do continente sul-americano e a cada reunião o sucesso se repetiu, com grande adesão de profissionais e estudantes. No IV EACS a quantidade de trabalhos em colaboração entre pesquisadores de diferentes países aumentou bastante em relação às edições anteriores. Isso significa que um dos objetivos principais dos EACS foi alcançado: o estreitamento das relações entre os membros da comunidade aracnológica sul-americana. Cabe destacar também que a qualidade dos trabalhos apresentados vem crescendo a cada edição do evento, colocando a pesquisa aracnológica sul-americana em um patamar cada vez mais elevado. A partir de 2005 será iniciada uma nova etapa com o "I Congresso Latino-Americano de Aracnologia", o qual esperamos em alguns anos também apresentar uma sinopse divulgando o sucesso do empreendimento.

\section{Agradecimentos}

Ao editor da Biota Neotropica e à FAPESP pela oportunidade de publicar os trabalhos do IV Encontro de Aracnólogos do Cone Sul. Aos colegas Miguel Simó, Arno Antonio Lise, Luis Eduardo Acosta e Ricardo Pinto da Rocha, organizadores dos EACS.

\section{Referências Bibliográficas}

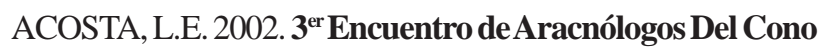
Sur. Programa y Resúmenes. Córdoba, Argentina, 110p.

ACOSTA, L.E. 2003. IV Encontro de Aracnólogos do Cone Sul. Revta Ibérica Aracnol., 8: 177-178.

AMERICANARACHNOLOGY. 2002. Reports from the $25^{\text {th }}$ annual meeting of the AAS - 7-11 July, 2001 Keene State College, Keene, New Hampshire. Newsletter Am. Arachnol. Soc., 64: 1-10.

CUSHING, P. 2003. Abstracts of the 2003 meeting at the Museum of Nature and Science in Denver. The American Arachnological Society, 60: Web version, http:// www.americanarachnology.org/MeetingAbstracts/ MeetingAbstractList.html 
DUNLOP, J. 2000. 18 ${ }^{\text {th }}$ European Colloquium of Arachnology, High Tatras, Slovakia, $12^{\text {th }}-17^{\text {th }}$ July 1999. Newsl. Br. Arachnol. Soc., 87: 4-6.

BELL, J.R. 2000. A meeting of arachnological minds: the $19^{\text {th }}$ European Colloquium of Arachnology, Ärhus, Denmark, $17^{\text {th}}-22^{\text {nd }}$ July 2000. Newsl. Br. Arachnol. Soc., 89: 1-3.

MACHADO, G. \& A.D. BRESCOVIT. 2003. IV Encontro de Aracnólogos do Cone Sul. São Pedro, São Paulo, 330p.

PENNEY, D. 2003. 21 $1^{\text {st }}$ European Colloquium of Arachnology, St Petesburg, Russia, $4^{\text {th }}-9^{\text {th }}$ August 2003. Newsl. Br. Arachnol. Soc., 98: 14-15.

PINTO-DA-ROCHA, R. 1996. Editorial. II Encontro de Aracnologia. 27/28 de abril de 1996 Serra do Japi - SP. Papo de Aranha, 2: 2-10.

PINTO-DA-ROCHA, R. 1997. Editorial. III Encontro de Aracnologia. 23/25 de maio de 1997 Serra do Japi - SP. Papo de Aranha, 4: 1-8.

REISKIND, J. 2003. Reports from the $26^{\text {th }}$ annual meeting Univ. of California - Riverside 25-29 June, 2002. Newsletter Am. Arachnol. Soc., 66: 1-13.

SHAW, E.M. 2002. 20 ${ }^{\text {th }}$ European Colloquium of Arachnology, Szombathely, Hungary, 22 ${ }^{\text {nd }}-26^{\text {th }}$ July 2002. Newsl. Br. Arachnol. Soc., 95: 1- 2.

STARR, C. 1999. Remarks and comments on the Trinidad Meeting. Newsletter of the American Arachnological Society, 60: Web version, http:// w w w. a m e ric a n a r c hnology.org / A A / AAS_news_Dec99.html\#starr

Título: Encontro de Aracnologia do Cone Sul (19972003): um investimento de sucesso.

Autores: Antonio D. Brescovit, Cristina Anne Rheims \& Glauco Machado

Biota Neotropica, Vol. 5 ( número 1a): 2005

http://www.biotaneotropica.org.br/v5n1a/pt/ abstract?point-of-view+bn022051a2005

Recebido em 07/12/2003 - Publicado em 01/02/2005

ISSN 1676-0603

http://www.biotaneotropica.org.br 